PROCEEDINGS OF THE

AMERICAN MATHEMATICAL SOCIETY

Volume 128, Number 11, Pages 3259-3268

S 0002-9939(00)05548-9

Article electronically published on April 28, 2000

\title{
FINITE REPRESENTABILITY OF OPERATORS IN THE SENSE OF BELLENOT
}

\author{
MANUELA BASALLOTE AND SANTIAGO DÍAZ-MADRIGAL \\ (Communicated by David R. Larson)
}

\begin{abstract}
We present several new results about the notion of finite representability of operators introduced by Bellenot.
\end{abstract}

\section{INTRODUCTION}

The theory of finite representability between Banach spaces has been considered by some authors in the more general context of operator theory. Mainly, three definitions have been proposed for the concept of finite representability between two operators and they are due to Beauzamy $\mathrm{B}]$, Bellenot [Be, and Heinrich [H2]. In this paper we are concerned with Bellenot's definition. We show that Bellenot's notion implies Beauzamy's notion in many situations but that they are far from being equivalent in spite of the line which divides these concepts is quite fine. As was pointed out in $[\mathrm{Be}$, there is no kind of relation between Bellenot's and Heinrich's concepts.

Bellenot's opinion about his definition was not very enthusiastic ("This is a strange definition, but it is what ..." [Be, p.7]). However, we present several results which show that, in our opinion, this is a useful and natural concept. Namely, we relate it with conmutative diagrams, we use it to characterize some operator ideals and we connect it with classical topics of linear algebra. We finish the paper giving an alternative proof of one of the main results of [Be, p.7]; that is, the biadjoint of every operator $T$ is finitely representable in $T$. We would like to mention that there seems to be a gap in the proof given there (see the proof of Condition (D) of Theorem 5 in [Be p.7]) but, anyway, as we have commented above, our approach is completely different.

In what follows, an operator will always mean a linear and continuous map. An operator $T: X \rightarrow Y$ between two Banach spaces is said to be an $\varepsilon$-isometry if

$$
\||\|T(x)\|-\|x\|| \leq \varepsilon\| x \|, \text { for all } x \in X,
$$

and $T$ is said to be a $(1+\varepsilon)$-isomorphism if $T$ is an isomorphism onto its image with $\|T\|\left\|T^{-1}\right\| \leq 1+\varepsilon$.

Received by the editors December 17, 1998.

1991 Mathematics Subject Classification. Primary 46B07, 46 B08.

This research has been partially supported by the DGICYT project no. PB97-0706 and by La Consejería de Educación y Ciencia de la Junta de Andalucía. 
The rest of our terminology and notations are quite standard and we refer the reader to [H1] for the theory of ultrapowers and to $\mathrm{Be}$ and $\mathrm{Di}$ for the theory of Banach spaces.

\section{Finite REPRESENTABILITy OF OPERATORS}

We begin by recalling the original definition of finite representability of operators introduced by Bellenot $\mathrm{Be}]$.

Definition 2.1. An operator $T_{0}: X_{0} \longrightarrow Y_{0}$ is said to be finitely representable in an operator $T: X \longrightarrow Y$ if, for every finite-dimensional subspace $M_{0} \subset X_{0}$ and $\varepsilon>0$, there is a linear map $V: M_{0} \rightarrow X$ such that

1. $V$ is an $\varepsilon$-isometry.

2. $\left|\left\|T_{0}(x)\right\|-\|T V(x)\|\right| \leq \varepsilon\|x\|$, for all $x \in M_{0}$.

Bellenot gave an additional third condition but he also noticed that it could be dropped. Recently, the authors (jointly with M. D. Contreras) have shown the following characterization of this notion $\mathrm{BCD}$.

Theorem 2.1. Let $T_{0}: X_{0} \rightarrow Y_{0}, T: X \rightarrow Y$ be two operators and consider the following statements:

(1) $T_{0}$ is finitely representable in $T$.

(2) There exist an ultrafilter $\mathcal{U}$ and two isometries $\mathcal{J}_{1}: X_{0} \rightarrow(X)_{\mathcal{U}}, \mathcal{J}_{2}: T_{0}\left(X_{0}\right)$ $\rightarrow(Y)_{\mathcal{U}}$ such that $\mathcal{J}_{2} T_{0}=(T)_{\mathcal{U}} \mathcal{J}_{1}$.

(3) For every finite-dimensional subspace $M_{0} \subset X_{0}$ and $\varepsilon>0$, there exist a finite-dimensional subspace $M \subset X$ and two $\varepsilon$-isometries $V: M_{0} \rightarrow M$ and $W: T_{0}\left(M_{0}\right) \rightarrow T(M)$ such that $W T_{0}=T V$.

Then, (1) $\Leftrightarrow(2)$ and, if $T_{0}$ is injective, (1) $\Leftrightarrow(2) \Leftrightarrow(3)$.

Beauzamy's definition of finite representability of operators appears just replacing the string " $\varepsilon$-isometries" by " $(1+\varepsilon)$-isomorphisms" in condition $(3)$ of the above theorem [B, p. 241]. Hence, Bellenot's concept implies Beauzamy's concept, whenever $T_{0}$ is injective. However, we are going to see that the converse is not true. At this point, we would like to stress that the usual computations used to pass from $(1+\varepsilon)$-isomorphisms to $\varepsilon^{\prime}$-isometries do not work in our case since they break the commutativity of the diagram.

Theorem 2.2. An operator $T: X \rightarrow Y$ is compact if and only if every operator finitely representable in $T$ is compact.

Proof. One implication is trivial. To obtain the other one, we are going to show that $(T)_{\mathcal{U}}$ is compact, for every ultrafilter $\mathcal{U}$ over a certain index set $I$.

Take $y=(T)_{\mathcal{U}}\left(x_{i}\right)_{\mathcal{U}}$, where $\left(x_{i}\right)_{\mathcal{U}} \in(X)_{\mathcal{U}}$ with $\left\|\left(x_{i}\right)_{\mathcal{U}}\right\| \leq 1$. Then, we can choose an element of the corresponding class $\left(x_{i}\right)_{i} \in \ell_{\infty}(I, X)$ and a set $I_{0} \in \mathcal{U}$ such that $\left\|x_{i}\right\| \leq 1$, for all $i \in I_{0}$. Since $T$ is compact, the following limit exists

$$
z:=\lim _{\mathcal{U}} T\left(x_{i}\right) \in \overline{T\left(B_{X}\right)}
$$

where $B_{X}$ is the closed unit ball of $X$. If $\mathcal{S}$ denotes the canonical injection of $Y$ into $(Y)_{\mathcal{U}}$, we have that

$$
\|y-\mathcal{S}(z)\|=\left\|\left(T x_{i}\right)_{\mathcal{U}}-\mathcal{S}(z)\right\|=\lim _{\mathcal{U}}\left\|T\left(x_{i}\right)-z\right\|=0 .
$$


Therefore, $\mathcal{S}(z)=y$ and we have shown that $(T) \mathcal{u}$ maps the closed unit ball of $(X)_{\mathcal{U}}$ into the compact set $\mathcal{S}\left(\overline{T\left(B_{X}\right)}\right)$. That is, $(T)_{\mathcal{U}}$ is compact.

Take an operator $T_{0}: X_{0} \rightarrow Y_{0}$ finitely representable in the compact operator $T$. By Theorem 2.1, we can find an ultrafilter $\mathcal{U}$ and two isometries $\mathcal{J}_{1}: X_{0} \rightarrow(X)_{\mathcal{U}}$, $\mathcal{J}_{2}: T_{0}\left(X_{0}\right) \rightarrow(Y)_{\mathcal{U}}$ such that $\mathcal{J}_{2} T_{0}=(T)_{\mathcal{U}} \mathcal{J}_{1}$. We know that $(T)_{\mathcal{U}}$ is compact. Since $\mathcal{J}_{2}$ is an isometry, we also have that $T_{0}$ is compact.

Example 2.1. Finite representability of operators in the sense of Beauzamy is a concept strictly wider than finite representability of operators in the sense of Bellenot.

Proof. Consider the Banach space $X:=\left(\bigoplus_{n=1}^{\infty} \ell_{q}^{n}\right)_{1}$, that is, the sum of the finite dimensional spaces $\ell_{q}^{n}$ with the $\ell_{1}$-norm $(1<q<\infty)$, and define the map $T: X \rightarrow$ $X$ given by

$$
\left(x_{n}\right) \in X \longmapsto T\left(x_{n}\right):=\left(\frac{1}{n} x_{n}\right) \in X .
$$

Clearly, $T$ is a well defined operator with $\|T\| \leq 1$. Moreover, $T$ is the limit in the operator norm of the sequence of operators $P_{N} T$, where $P_{N}$ is the projection from $X$ onto the first $N$ coordinates. Since $P_{N} T$ have finite rank, we deduce that $T$ is compact. On the other hand, denote by $T_{0}$ the identity operator on the Banach space $\ell_{q}(1<q<\infty)$.

We notice that $T_{0}$ is not finitely representable in $T$ in the sense of Bellenot. Otherwise, using that $T$ is compact and according to Theorem 2.2, we obtain that $T_{0}$ is compact and, therefore, $\ell_{q}$ is finite dimensional.

Now, we are going to see that $T_{0}$ is finitely representable in $T$ in the sense of Beauzamy. Let $\varepsilon>0$ and $M_{0}$ be a finite-dimensional subspace of $\ell_{q}$. Take $\varepsilon^{\prime}>0$ such that $\left(1+\varepsilon^{\prime}\right)\left(1-\varepsilon^{\prime}\right)^{-1} \leq 1+\varepsilon$ and $0<\varepsilon^{\prime}<\min \{1, \varepsilon\}$.

Since $\ell_{q}$ is a $\mathcal{L}_{q}$-space, we can find an $\varepsilon^{\prime}$-isometry $V_{1}: M_{0} \rightarrow \ell_{q}^{N}$, for some $N \in \mathbb{N}$. If $J$ is the canonical injection of $\ell_{q}^{N}$ into $\left(\bigoplus_{n=1}^{\infty} \ell_{q}^{n}\right)_{1}$, we consider $V:=J V_{1}$. Obviously, $V$ is an $\varepsilon$-isometry.

Define $W: T_{0}\left(M_{0}\right)=M_{0} \rightarrow X$ by

$$
W(m)=\left(0,0, \ldots, 0, \frac{1}{N} V_{1}(m)\{\text { the } N \text {-th position }\}, 0, \ldots\right), \quad m \in M_{0} .
$$

Obviously, $W$ is an isomorphism onto its image and $W T_{0}=T V$. Moreover,

$$
\|W\| \leq \frac{1}{N}\left\|V_{1}\right\| \leq \frac{1+\varepsilon^{\prime}}{N}, \quad\left\|W^{-1}\right\| \leq N\left\|V^{-1}\right\| \leq \frac{N}{1-\varepsilon^{\prime}} .
$$

Therefore

$$
\|W\|\left\|W^{-1}\right\| \leq \frac{1+\varepsilon^{\prime}}{1-\varepsilon^{\prime}} \leq 1+\varepsilon
$$

and $W$ is a $(1+\varepsilon)$-isomorphism.

We have seen that Bellenot's concept can be characterized by commutative diagrams and ultrapowers. The next theorem gives another result of this type. Roughly speaking, the diagram from Theorem 2.1(3) can be considered the "injective diagram" and the next one is the "surjective diagram".

We recall that, if $T: X \rightarrow Y$ is a surjective operator, then open $(T)$ denotes the infimum of the constants $M>0$ such that, for every $y \in Y$, there is $x \in X$ with

$$
T x=y \text {, and }\|x\| \leq M\|y\| .
$$


Theorem 2.3. Let $T_{0}: X_{0} \rightarrow Y_{0}$ and $T: X \rightarrow Y$ be operators with $T_{0}$ surjective. If $T_{0}^{*}$ is finitely representable in $T^{*}$, then there exist an ultrafilter $\mathcal{U}$ and two surjective operators $Q_{1}:(Y)_{\mathcal{U}} \rightarrow Y_{0}^{* *}$ and $Q_{2}:(X)_{\mathcal{U}} \rightarrow\left(\left(T_{0}^{*}\left(Y_{0}^{*}\right)\right)^{*}\right.$ with

$$
\left\|Q_{1}\right\| \leq 1,\left\|Q_{2}\right\| \leq 1, \operatorname{open}\left(Q_{1}\right) \leq 1, \text { open }\left(Q_{2}\right) \leq 1,
$$

and $Q_{1}(T)_{\mathcal{U}}=T_{0}^{* *} Q_{2}$.

Proof. Let $\mathcal{U}$ be an ultrafilter dominating the canonical order filter defined on the set $\mathcal{I}$ of all pairs $\left(M_{0}, \varepsilon\right)$ with $M_{0}$ a finite-dimensional subspace of $Y_{0}^{*}$ and $0<\varepsilon<1 / 2$. By Theorem 2.1, for each $i=\left(M_{0}^{i}, \varepsilon_{i}\right) \in \mathcal{I}$, there exist a finite-dimensional subspace $M^{i}$ of $Y^{*}$ and two $\varepsilon_{i}$-isometries, $V_{i}: M_{0}^{i} \longrightarrow M^{i}$ and $W_{i}: T_{0}^{*}\left(M_{0}^{i}\right) \longrightarrow T^{*}\left(M^{i}\right)$ such that $W_{i} T_{0}^{*}=T^{*} V_{i}$. Now, for each $i \in \mathcal{I}$ and $y_{0}^{*} \in Y_{0}^{*}$, we define the element of $Y^{*}$ given by

$$
\mathcal{J}_{i}\left(y_{0}^{*}\right)=\left\{\begin{array}{lll}
V_{i}\left(y_{0}^{*}\right) & \text { if } & y_{0}^{*} \in M_{0}^{i} \\
0 & \text { if } & y_{0}^{*} \notin M_{0}^{i}
\end{array}\right.
$$

and consider the following two maps:

$$
\begin{gathered}
\mathcal{J}_{1}: Y_{0}^{*} \rightarrow\left(Y^{*}\right) \mathcal{U}, \quad y_{0}^{*} \mapsto \mathcal{J}_{1}\left(y_{0}^{*}\right):=\left(\mathcal{J}_{i}\left(y_{0}^{*}\right)\right) \mathcal{U}, \\
\mathcal{J}_{2}: T_{0}^{*}\left(Y_{0}^{*}\right) \rightarrow\left(X^{*}\right) \mathcal{U}, \quad T_{0}^{*}\left(y_{0}^{*}\right) \mapsto \mathcal{J}_{2}\left(T_{0}^{*}\left(y_{0}^{*}\right)\right):=\left(T^{*}\right) \mathcal{U} \mathcal{J}_{1}\left(y_{0}^{*}\right)=\left(T^{*} \mathcal{J}_{i}\left(y_{0}^{*}\right)\right)_{\mathcal{U}}
\end{gathered}
$$

It is not difficult to see that $\mathcal{J}_{1}$ and $\mathcal{J}_{2}$ are (well defined) linear isometries. It is well-known that the Banach spaces $(Y)_{\mathcal{U}}$ and $(X)_{\mathcal{U}}$ can be canonically placed in $\left(\left(Y^{*}\right) \mathcal{U}\right)^{*}$ and $\left(\left(X^{*}\right) \mathcal{U}\right)^{*}$, respectively. At this stage, we define $Q_{1}$ as the restriction of $\mathcal{J}_{1}^{*}$ to $(Y)_{\mathcal{U}}$ and $Q_{2}$ as the restriction of $\mathcal{J}_{2}^{*}$ to $(X)_{\mathcal{U}}$. Clearly,

$$
\left\|Q_{1}\right\| \leq 1,\left\|Q_{2}\right\| \leq 1, \quad \text { and } \quad Q_{1}(T)_{\mathcal{U}}=T_{0}^{* *} Q_{2} .
$$

Let us show that $Q_{1}$ is surjective and open $\left(Q_{1}\right) \leq 1$. Given $y_{0}^{* *} \in Y_{0}^{* *}$ and, for each $i=\left(M_{0}^{i}, \varepsilon_{i}\right) \in \mathcal{I}$, we denote the restriction of $y_{0}^{* *}$ to $M_{0}^{i}$ by $z_{i}$. We have that $z_{i} \in\left(M_{0}^{i}\right)^{*}$ and $\left(V_{i}^{*}\right)^{-1} z_{i} \in\left(M^{i}\right)^{*}$. Bearing in mind the isometric identification,

$$
\left(M^{i}\right)^{*} \equiv Y /\left(M^{i}\right)_{\perp},\left(\text { where }\left(M^{i}\right)_{\perp} \text { is the orthogonal subset of } M \text { in } Y\right),
$$

there is $y_{i} \in Y$ such that

$$
y_{i}+\left(M^{i}\right)_{\perp} \equiv\left(V_{i}^{*}\right)^{-1} z_{i} \text { and }\left\|y_{i}\right\| \leq\left\|\left(V_{i}^{*}\right)^{-1} z_{i}\right\|+\varepsilon_{i} .
$$

For each $i \in \mathcal{I}$,

$$
\left\|y_{i}\right\| \leq\left\|\left(V_{i}^{*}\right)^{-1} z_{i}\right\|+\varepsilon_{i} \leq \frac{1}{1-\varepsilon_{i}}\left\|y_{0}^{* *}\right\|+\varepsilon_{i} \leq 2\left\|y_{0}^{* *}\right\|+1 .
$$

Therefore, we can consider the element $\left(y_{i}\right)_{\mathcal{U}} \in(Y)_{\mathcal{U}}$ and we have

$$
\left\|\left(y_{i}\right) \mathcal{U}\right\| \leq \lim _{\mathcal{U}}\left(\frac{1}{1-\varepsilon_{i}}\left\|y_{0}^{* *}\right\|+\varepsilon_{i}\right) \leq\left\|y_{0}^{* *}\right\| .
$$

Moreover, we are going to see that $Q_{1}\left(\left(y_{i}\right)_{\mathcal{U}}\right)=y_{0}^{* *}$. Given $y_{0}^{*} \in Y_{0}^{*}$ and using the definition of $\mathcal{J}_{1}$, we have that

$$
\left\langle Q_{1}\left(\left(y_{i}\right)_{\mathcal{U}}\right), y_{0}^{*}\right\rangle=\left\langle\left(y_{i}\right)_{\mathcal{U}}, \mathcal{J}_{1}\left(y_{0}^{*}\right)\right\rangle=\left\langle\left(y_{i}\right)_{\mathcal{U}},\left(\mathcal{J}_{i}\left(y_{0}^{*}\right)\right)_{\mathcal{U}}\right\rangle=\lim _{\mathcal{U}}\left\langle y_{i}, \mathcal{J}_{i}\left(y_{0}^{*}\right)\right\rangle .
$$

For each $i=\left(M_{0}^{i}, \varepsilon_{i}\right) \in \mathcal{I}$, we consider the projection $P_{i}: Y_{0}^{*} \rightarrow M_{0}^{i}$. It is not difficult to check that

$$
\lim _{\mathcal{U}}\left\langle y_{i}, \mathcal{J}_{i}\left(y_{0}^{*}\right)\right\rangle=\lim _{\mathcal{U}}\left\langle y_{i}, V_{i} P_{i}\left(y_{0}^{*}\right)\right\rangle
$$


Then, bearing in mind that $V_{i} P_{i}\left(y_{0}^{*}\right) \in M^{i}$, we obtain that

$$
\begin{aligned}
\left\langle Q_{1}\left(\left(y_{i}\right)_{\mathcal{U}}\right), y_{0}^{*}\right\rangle & =\lim _{\mathcal{U}}\left\langle y_{i}+\left(M^{i}\right)_{\perp}, V_{i} P_{i}\left(y_{0}^{*}\right)\right\rangle=\lim _{\mathcal{U}}\left\langle\left(V_{i}^{*}\right)^{-1} z_{i}, V_{i} P_{i}\left(y_{0}^{*}\right)\right\rangle \\
& =\lim _{\mathcal{U}}\left\langle z_{i}, P_{i}\left(y_{0}^{*}\right)\right\rangle=\lim _{\mathcal{U}}\left\langle y_{0}^{* *}, P_{i}\left(y_{0}^{*}\right)\right\rangle=\left\langle y_{0}^{* *}, y_{0}^{*}\right\rangle .
\end{aligned}
$$

It remains to prove that $Q_{2}$ is also surjective with open $\left(Q_{2}\right) \leq 1$. Given $x_{0}^{* *} \in$ $\left(T_{0}^{*}\left(Y_{0}^{*}\right)\right)^{*}$ and, by Hahn-Banach's theorem, we extend it to the whole space $X_{0}^{*}$ with the same norm and we denote this extension by $z_{0}^{* *} \in X_{0}^{* *}$. For each $i=$ $\left(M_{0}^{i}, \varepsilon_{i}\right) \in \mathcal{I}$, we also denote the restriction of $z_{0}^{* *}$ to $T_{0}^{*}\left(M_{0}^{i}\right)$ by $z_{i}$. We have $\left(W_{i}^{*}\right)^{-1} z_{i} \in\left(T^{*}\left(M^{i}\right)\right)^{*}$ and there is $x_{i} \in X$ such that

$$
x_{i}+\left(T^{*}\left(M^{i}\right)\right)_{\perp} \equiv\left(W_{i}^{*}\right)^{-1} z_{i} \quad \text { and } \quad\left\|x_{i}\right\| \leq\left\|\left(W_{i}^{*}\right)^{-1} z_{i}\right\|+\varepsilon_{i}
$$

$\left(\left(T^{*}\left(M^{i}\right)\right)_{\perp}\right.$ is the orthogonal of $T^{*}\left(M^{i}\right)$ in $\left.X\right)$. Then,

$$
\left\|x_{i}\right\| \leq\left\|\left(W_{i}^{*}\right)^{-1} z_{i}\right\|+\varepsilon_{i} \leq \frac{1}{1-\varepsilon_{i}}\left\|x_{0}^{* *}\right\|+\varepsilon_{i} \leq 2\left\|x_{0}^{* *}\right\|+1
$$

and we can consider the element $\left(x_{i}\right)_{\mathcal{U}} \in(X)_{\mathcal{U}}$. We notice that

$$
\left\|\left(x_{i}\right) \mathcal{U}\right\| \leq \lim \left(\frac{1}{1-\varepsilon_{i}}\left\|x_{0}^{* *}\right\|+\varepsilon_{i}\right)=\left\|x_{0}^{* *}\right\| .
$$

Moreover, we are going to see that $Q_{2}\left(\left(x_{i}\right)_{\mathcal{U}}\right)=x_{0}^{* *}$. Given $T_{0}^{*}\left(y_{0}^{*}\right) \in T_{0}^{*}\left(Y_{0}^{*}\right)$, we have that

$$
\begin{aligned}
\left\langle Q_{2}\left(\left(x_{i}\right) \mathcal{U}\right), T_{0}^{*}\left(y_{0}^{*}\right)\right\rangle & =\left\langle\left(x_{i}\right) \mathcal{U}, \mathcal{J}_{2} T_{0}^{*}\left(y_{0}^{*}\right)\right\rangle=\left\langle\left(x_{i}\right) \mathcal{U},\left(T^{*} \mathcal{J}_{i}\left(y_{0}^{*}\right)\right) \mathcal{U}\right\rangle \\
& =\lim _{\mathcal{U}}\left\langle x_{i}, T^{*} \mathcal{J}_{i}\left(y_{0}^{*}\right)\right\rangle=\lim _{\mathcal{U}}\left\langle x_{i}, T^{*} V_{i} P_{i}\left(y_{0}^{*}\right)\right\rangle .
\end{aligned}
$$

Bearing in mind that $T^{*} V_{i} P_{i}\left(y_{0}^{*}\right) \in T^{*}\left(M^{i}\right)$, we deduce

$$
\begin{aligned}
\left\langle Q_{2}\left(\left(x_{i}\right) \mathcal{U}\right), T_{0}^{*}\left(y_{0}^{*}\right)\right\rangle & =\lim _{\mathcal{U}}\left\langle x_{i}+\left(T^{*} M^{i}\right)_{\perp}, T^{*} V_{i} P_{i}\left(y_{0}^{*}\right)\right\rangle \\
& =\lim _{\mathcal{U}}\left\langle\left(W_{i}^{*}\right)^{-1} z_{i}, W_{i} T_{0}^{*} P_{i}\left(y_{0}^{*}\right)\right\rangle=\lim _{\mathcal{U}}\left\langle z_{i}, T_{0}^{*} P_{i}\left(y_{0}^{*}\right)\right\rangle \\
& =\lim _{\mathcal{U}}\left\langle z_{0}^{* *}, T_{0}^{*} P_{i}\left(y_{0}^{*}\right)\right\rangle=\left\langle x_{0}^{* *}, T_{0}^{*}\left(y_{0}^{*}\right)\right\rangle .
\end{aligned}
$$

Beauzamy characterized the class of uniformly convexifying operators through his concept of finite representability. Heinrich's monograph [H2] also pays special attention to this kind of topic. In the same way, we are going to see that several classes of operators can be characterized with Bellenot's concept (see also Theorem $2.2)$.

We refer the reader to $[\vec{B}$ for the definitions of uniformly convexifying, Rademacher cotype and Rademacher type operators. But, we recall that an operator $T: X \rightarrow Y$ is said to be tauberian if the kernel of $T+K$ is reflexive, for all compact operators $K: X \rightarrow Y$, and supertauberian if the kernel of $T+K$ is super-reflexive, for all compact operators $K: X \rightarrow Y$.

Theorem 2.4. Let $T: X \rightarrow Y$ be an operator.

(1) Every operator finitely representable in $T$ is weakly compact if and only if $T$ is uniformly convexifying.

(2) Every operator finitely representable in $T$ is weakly conditionally compact if and only if $T$ is of Rademacher type. 
(3) Every operator finitely representable in $T$ is unconditionally convergent if and only if $T$ is of Rademacher cotype.

(4) Every operator finitely representable in $T$ is tauberian if and only if $T$ is supertauberian.

Proof. The first two statements are implicitely given in $[\mathrm{BCD}$, so we omit the proof. The third statement follows easily from Theorem 2.1 and the following two facts: an operator is unconditionally convergent if and only if it does not fix a copy of $c_{0}$ [Di]; an operator $S$ is of Rademacher cotype if and only if, for every ultrafilter $\mathcal{U},(S)_{\mathcal{U}}$ does not fix a copy of $c_{0}[\mathrm{BCD}$.

$(4)(\Rightarrow)$ Assume that $T_{0}: X_{0} \rightarrow Y_{0}$ is finitely representable in $T$ and suppose that $T_{0}$ is not tauberian. By $\mathrm{GA}$, there is $0<\varepsilon<1$ such that, for every $\delta>0$ and every $n \in \mathbb{N}$, we can find $x_{0}(1), \ldots, x_{0}(n)$ in the closed unit ball of $X_{0}$ and $x_{0}^{*}(1), \ldots, x_{0}^{*}(n)$ in the closed unit ball of $X_{0}^{*}$ such that

$$
\begin{array}{lll}
\left\langle x_{0}^{*}(k), x_{0}(l)\right\rangle>\varepsilon & \text { if } & 1 \leq k \leq l \leq n, \\
\left\langle x_{0}^{*}(k), x_{0}(l)\right\rangle=0 & \text { if } & 1 \leq l<k \leq n, \\
\left\|T_{0}\left(x_{0}(k)\right)\right\|<\delta, & k=1, \ldots, n .
\end{array}
$$

Consider the finite-dimensional subspace of $X_{0}$

$$
M_{0}:=\operatorname{span}\left\{x_{0}(1), \ldots, x_{0}(n)\right\} .
$$

Since $T_{0}$ is finitely representable in $T$, by Theorem 2.1 , there exist finite-dimensional subspace $M$ of $X$ and a $\delta$-isometry $V: M_{0} \rightarrow M$ such that $\|T V(x)\| \leq\left\|T_{0}(x)\right\|+$ $\delta\|x\|$. Let us consider $V^{\prime}:=\frac{1}{\|V\|} V$. For each $k=1, \ldots, n$, we define the linear functional $y_{k}^{*}: M \rightarrow \mathbb{K}$ by

$$
y_{k}^{*}(x):=\left\langle x_{0}^{*}(k),\left(V^{\prime}\right)^{-1}(x)\right\rangle, \quad x \in M .
$$

We have

$$
\left\|y_{k}^{*}\right\| \leq\left\|\left(V^{\prime}\right)^{-1}\right\|\left\|x_{0}^{*}(k)\right\| \leq \frac{1+\delta}{1-\delta} \leq 3 .
$$

By Hahn-Banach's theorem, we extend $y_{k}^{*}$ to $X$ with the same norm and we still denote this extension by $y_{k}^{*}$.

Now, for each $n \in \mathbb{N}$ and $\delta>0$, we consider the following two finite sets:

$$
A(n, \delta):=\left\{V^{\prime}\left(x_{0}(1)\right), \ldots, V^{\prime}\left(x_{0}(n)\right)\right\}, \quad B(n, \delta):=\left\{\frac{1}{3} y_{1}^{*}, \ldots, \frac{1}{3} y_{n}^{*}\right\} .
$$

Clearly, $A(n, \delta)$ and $B(n, \delta)$ are contained in the closed unit ball of $X$ and $X^{*}$, respectively. Moreover, we have that, if $1 \leq k \leq l \leq n$,

$$
\left\langle\frac{1}{3} y_{k}^{*}, V^{\prime}\left(x_{0}(l)\right)\right\rangle=\frac{1}{3}\left\langle x_{0}^{*}(k), x_{0}(l)\right\rangle>\frac{\varepsilon}{3},
$$

if $1 \leq l<k \leq n$,

$$
\left\langle\frac{1}{3} y_{k}^{*}, V^{\prime}\left(x_{0}(l)\right)\right\rangle=\frac{1}{3}\left\langle x_{0}^{*}(k), x_{0}(l)\right\rangle=0,
$$

and, for each $k=1, \ldots, n$,

$$
\left\|T V^{\prime}\left(x_{0}(k)\right)\right\|=\frac{1}{\|V\|}\left(\left\|T_{0}\left(x_{0}(k)\right)\right\|+\delta\right) \leq \frac{2 \delta}{1-\delta} .
$$

According to [GA], $T$ is not supertauberian and we get a contradiction. 
$(4)(\Leftarrow)$ Let $\mathcal{U}$ be an ultrafilter over $\mathbb{N}$. By Theorem 2.1 , we see that $(T)_{\mathcal{U}}$ is finitely representable in $T$, so $(T)_{\mathcal{U}}$ is a tauberian operator. Then, by GA], we conclude that $T$ is supertauberian.

Our next result shows that there are cases where Bellenot's definition can be rewritten in very classical terms. Namely, the corollary to the next proposition is a version of the well-known "singular value decomposition theorem" of linear algebra.

Proposition 2.5. Let $A$ and $B$ be real matrices, both of dimensions $k \times n$, viewed as operators from $\ell_{2}^{n}$ into $\ell_{2}^{k}$. Then, $A$ is finitely representable in $B$ if and only if there are two orthogonal matrices $P$ and $Q$ such that $B=P A Q^{t}$.

Proof. $(\Leftarrow)$ Given a finite-dimensional subspace $M_{0}$ of $\ell_{2}^{n}$ and $\varepsilon>0$, we define $V$ (resp. $W$ ) as the restriction to $M_{0}$ (resp. to $A\left(M_{0}\right)$ ) of the isometry induced in $\ell_{2}^{n}$ (resp. in $\ell_{2}^{k}$ ) by the matrix $P$ (resp. $Q$ ). It is trivial that $V$ and $W$ are $(1+\varepsilon)-$ isometries and $V A=B W$. Therefore, by Theorem 2.1, $A$ is finitely representable in $B$.

$(\Rightarrow)$ In this implication, we denote by $T_{A}$ and $T_{B}$ the operators from $\ell_{2}^{n}$ into $\ell_{2}^{k}$ induced by $A$ and $B$, respectively. We may assume that $A$ is not the null matrix, so the dimension of $T_{A}\left(\ell_{2}^{n}\right)$ is positive. Since $T_{A}$ is finitely represented in $T_{B}$ and again, by Theorem 2.1, for each natural number $m>2$, we can find two $\frac{1}{m}$-isometries

$$
V_{m}: \ell_{2}^{n} \rightarrow \ell_{2}^{n}, \quad W_{m}: T_{A}\left(\ell_{2}^{n}\right) \rightarrow T_{B}\left(\ell_{2}^{n}\right)
$$

such that $W_{m} T_{A}=T_{B} V_{m}$. We notice that this equality says that $W_{m}$ is surjective, for all $m$.

Obviously, $\left(V_{m}\right)$ is a bounded sequence of the Banach space $B\left(\ell_{2}^{n}\right)$ of all operators from $\ell_{2}^{n}$ into $\ell_{2}^{n}$. Since $B\left(\ell_{2}^{n}\right)$ is a finite-dimensional Banach space, we can extract a subsequence $\left(V_{m_{l}}\right)$ of $\left(V_{m}\right)$ such that the sequence $\left(V_{m_{l}}\right)$ converges to some $V \in$ $B\left(\ell_{2}^{n}\right)$. Clearly, $V$ is an isometry in $\ell_{2}^{n}$. By a similar argument, we may, and do, assume that the sequence $\left(W_{m_{l}}\right)$ converges in the operator norm to some (surjective) isometry $W: T_{A}\left(\ell_{2}^{n}\right) \rightarrow T_{B}\left(\ell_{2}^{n}\right)$.

Take an orthonormal basis $\left\{f_{1}, \ldots, f_{r}\right\}(1 \leq r \leq k)$ of $T_{A}\left(\ell_{2}^{n}\right) \subset \ell_{2}^{k}$. Since $W$ is a surjective isometry, $\left\{W\left(f_{1}\right), \ldots, W\left(f_{r}\right)\right\}$ is also an orthonormal basis of $T_{B}\left(\ell_{2}^{n}\right)$. We extend these bases to the following two orthonormal bases of $\ell_{2}^{k}$,

$$
B_{1}:=\left\{f_{1}, \ldots, f_{r}, f_{r+1}, \ldots, f_{k}\right\} \text { and } B_{2}:=\left\{W\left(f_{1}\right), \ldots, W\left(f_{r}\right), g_{r+1}, \ldots, g_{k}\right\}
$$

Then, we define the linear map $\widetilde{W}: \ell_{2}^{k} \rightarrow \ell_{2}^{k}$ by

$$
\sum_{i=1}^{k} \lambda_{i} f_{i} \longmapsto \widetilde{W}\left(\sum_{i=1}^{k} \lambda_{i} f_{i}\right):=W\left(\sum_{i=1}^{r} \lambda_{i} f_{i}\right)+\sum_{i=r+1}^{k} \lambda_{i} g_{i} .
$$

It is clear that we still have $\widetilde{W} T_{A}=T_{B} V$.

At this point, let $C_{n}$ and $C_{k}$ be the canonical basis of $\ell_{2}^{n}$ and $\ell_{2}^{k}$, respectively. It is well known that $A$ and $B$ are the matrices which represent $T_{A}$ and $T_{B}$ with respect to $C_{n}-C_{k}$. On the other hand, let $Q$ be the matrix associated to $V$ with respect to $C_{n}-C_{n}$. Since $V$ is an isometry in $\ell_{2}^{n}$, we have that $Q$ is an orthogonal matrix of order $n$. By elementary linear algebra, we deduce that $B Q=P A$, where $P$ is the matrix associated to $\widetilde{W}$ with respect to $C_{k}-C_{k}$. 
Now, let $i d$ be the identity in $\ell_{2}^{k}, P_{1}$ be the matrix associated to $i d$ with respect to $C_{k}-B_{1}$ and $P_{2}$ be the matrix associated to $i d$ with respect to $B_{2}-C_{k}$. Therefore,

$$
P=P_{2}[\text { Identity matrix of order } k] P_{1} \text {. }
$$

Moreover, since both of $P_{1}$ and $P_{2}$ are orthogonal, we deduce that $P$ is orthogonal and this concludes the proof.

Corollary 2.6. Every real square matrix $A$ is finitely representable in the sense of Bellenot in a diagonal matrix whose entries are the singular values of $A$.

Finally, we present an alternative proof of one of the basic results of $[\mathrm{Be}]$ which can be seen as a variant in operator theory of the famous "principle of local reflexivity". As we commented in the introduction, there seems to be a gap in the proof given in $\mathrm{Be}$ p.7].

Theorem 2.7. The biadjoint of every operator $T: X \longrightarrow Y$ is finitely representable in $T$.

Proof. Let $M$ be a finite-dimensional subspace of $X^{* *}$ and $\varepsilon>0$. Fix $\delta>0$ and consider a $\delta / 2$-net $\left\{m_{1}, \ldots, m_{n}\right\}$ in the unit sphere of $M$. For each $k \in\{1, \ldots, n\}$, take an element $x_{k}^{*}$ of the unit sphere of $X^{*}$ such that $\left|\left\langle m_{k}, x_{k}^{*}\right\rangle\right|+\delta / 2>1$. If we define $F_{0}:=\operatorname{span}\left\{x_{1}^{*}, \ldots, x_{n}^{*}\right\}$, we have that

$$
(1-\delta)\|m\| \leq \sup \left\{\left|\left\langle m, f_{0}\right\rangle\right|: f_{0} \in F_{0},\left\|f_{0}\right\|=1\right\}, \quad \text { for all } m \in M .
$$

Now, we consider a $\delta / 2$-net $\left\{h_{1}, \ldots, h_{r}\right\}$ in the unit sphere of (the finite-dimensional space) $T^{* *}(M)$. For each $k \in\{1, \ldots, r\}$, take an element $y_{k}^{*}$ in the unit sphere of $Y^{*}$ such that $\left|\left\langle h_{k}, y_{k}^{*}\right\rangle\right|+\delta / 2>1$. If we define $F_{1}:=\operatorname{span}\left\{y_{1}^{*}, \ldots, y_{r}^{*}\right\}$, we have that

$$
(1-\delta)\left\|T^{* *}(m)\right\| \leq \sup \left\{\left|\left\langle T^{* *}(m), f_{1}\right\rangle\right|: f_{1} \in F_{1},\left\|f_{1}\right\|=1\right\}, \quad \text { for all } m \in M \text {. }
$$

Let $B(M, X)$ be the Banach space of all linear and continuous operators from $M$ to $X$. Given $m \in M$ and $x^{*} \in X^{*}$, we denote by $\phi\left(m, x^{*}\right)$ the element of the dual of $B(M, X)$ defined by

$$
\left\langle\phi\left(m, x^{*}\right), T\right\rangle=\left\langle T(m), x^{*}\right\rangle, \quad T \in B(M, X) .
$$

Put

$$
F:=\operatorname{span}\left\{\phi\left(m, f_{0}\right), \phi\left(m, T^{*} f_{1}\right): m \in M, f_{0} \in F_{0}, f_{1} \in F_{1}\right\} \subset B(M, X)^{*},
$$

and let $I$ be the formal inclusion of $M$ into $X^{* *}$ viewed as an element of the bidual of $B(M, X)[\mathrm{D}$. Then, by Helly's lemma and, for each finite-dimensional subspace $G \subset(B(M, X))^{*}$ containing $F$, we can find an operator $S_{G}: M \rightarrow X$ such that $\left\langle g, S_{G}\right\rangle=\langle I, g\rangle$, for every $g \in G$ and $\left\|S_{G}\right\| \leq(1+\delta)\|I\| \leq 1+\delta$.

Therefore, for every $m \in M$, we have

$$
\begin{aligned}
(1-\delta)\|m\| & \leq \sup \left\{\left|\left\langle m, f_{0}\right\rangle\right|: f_{0} \in F_{0},\left\|f_{0}\right\|=1\right\} \\
& =\sup \left\{\left|\left\langle I, \phi\left(m, f_{0}\right)\right\rangle\right|: f_{0} \in F_{0},\left\|f_{0}\right\|=1\right\} \\
& =\sup \left\{\left|\left\langle S_{G}(m), f_{0}\right\rangle\right|: f_{0} \in F_{0},\left\|f_{0}\right\|=1\right\} \\
& \leq\left\|S_{G}(m)\right\| \leq(1+\delta)\|m\| .
\end{aligned}
$$

That is, $S_{G}$ is an $\delta$-isometry, for every $G$. A similar argument shows that, for every $G$,

$$
(1-\delta)\left\|T^{* *}(m)\right\| \leq\left\|T S_{G}(m)\right\|, \text { for all } m \in M
$$


That is, we have shown that, for every $\delta>0$ and every finite-dimensional subspace $G \subset(B(M, X))^{*}$ containing $F$, the subset $C_{\delta}^{G} \subset B(M, X)$ is not empty, where $C_{\delta}^{G}$ is formed by all $\dot{\delta}$-isometries $S$ from $M$ to $X$ satisfying that

$$
\begin{gathered}
\langle g, S\rangle=\langle I, g\rangle, \quad g \in G, \\
(1-\delta)\left\|T^{* *}(m)\right\| \leq\|T S(m)\|, \quad m \in M .
\end{gathered}
$$

Define $C_{\delta}:=\bigcup\left\{C_{\delta}^{G}: G \subset(B(M, X))^{*}, G \supset F\right.$ and $\left.\operatorname{dim} G<\infty\right\}$. Looking at the subspaces $F_{0}$ and $F_{1}$, it is not difficult to see that $C_{\delta}$ is a convex subset of $B(M, X)$. Moreover, let us consider the following subsets of $Y^{n}$ :

$$
\begin{gathered}
D:=\left\{\left(y_{1}, \ldots, y_{n}\right) \in Y^{n}:\left\|y_{k}\right\|<\left\|T^{* *}\left(m_{k}\right)\right\|+\delta, k=1, \ldots, n\right\}, \\
\widetilde{C_{\delta}}:=\left\{\left(T S\left(m_{1}\right), \ldots, T S\left(m_{n}\right)\right) \in Y^{n}: S \in C_{\delta}\right\} .
\end{gathered}
$$

Clearly, $D$ is a non-empty convex open subset of $Y^{n}$ and $\widetilde{C_{\delta}}$ is a non-empty convex subset of $Y^{n}$. We claim that $D \cap \widetilde{C_{\delta}} \neq \emptyset$. Therefore, there is an $\delta$-isometry $S: M \rightarrow$ $X$ such that

$$
\begin{gathered}
(1-\delta)\left\|T^{* *}(m)\right\| \leq\|T S(m)\|, \quad \text { for all } m \in M, \\
\left\|T S\left(m_{k}\right)\right\| \leq\left\|T^{* *}\left(m_{k}\right)\right\|+\delta, \quad k=1, \ldots, n .
\end{gathered}
$$

Using that $\left\{m_{1}, \ldots, m_{n}\right\}$ is a $\delta / 2$-net in the unit sphere of $M$, we deduce that

$$
\|T S(m)\| \leq\left\|T^{* *}(m)\right\|+\delta+\delta\|T\|+\frac{\delta}{2}(1+\delta)\|T\|, \quad \text { for all } m \in M
$$

Hence, according to Definition 2.1, if we choose $\delta>0$ such that

$$
\delta+\delta\|T\|+\frac{\delta}{2}(1+\delta)\|T\|<\varepsilon \text { and } \delta<\varepsilon,
$$

we have that $T^{* *}$ is finitely representable in $T$ in the sense of Bellenot.

Now, we are going to prove the claim. If $D \cap \widetilde{C_{\delta}}=\emptyset$, by Hahn-Banach's theorem, we can find $\alpha \in \mathbb{R}$ and $g=\left(g_{1}, \ldots, g_{n}\right) \in\left(Y^{*}\right)^{n} \equiv\left(Y^{n}\right)^{*}$ such that

$$
\operatorname{Re}\left\langle g, y_{1}\right\rangle<\alpha \leq \operatorname{Re}\left\langle g, y_{2}\right\rangle, \quad \text { for all } y_{1} \in D, y_{2} \in \widetilde{C_{\delta}} \text {. }
$$

We see that $g \neq 0$. Consider the space $G$ formed by the linear hull of the set

$$
\left\{\phi\left(m, f_{0}\right), \phi\left(m, T^{*}\left(f_{1}\right)\right), \phi\left(m, T^{*}\left(g_{k}\right)\right): k=1, \ldots, n, m \in M, f_{0} \in F_{0}, f_{1} \in F_{1}\right\} .
$$

The corresponding operator $S_{G} \in C_{\delta}$ satisfies that

$$
\begin{aligned}
\alpha & \leq \operatorname{Re}\left\langle g,\left(T S_{G}\left(m_{1}\right), \ldots, T S_{G}\left(m_{n}\right)\right)\right\rangle=\operatorname{Re}\left(\sum_{k=1}^{n}\left\langle T^{*}\left(g_{k}\right), S_{G}\left(m_{k}\right)\right\rangle\right) \\
& =\operatorname{Re}\left(\sum_{k=1}^{n}\left\langle g_{k}, T^{* *}\left(m_{k}\right)\right\rangle\right)=\operatorname{Re}\left\langle g,\left(T^{* *}\left(m_{1}\right), \ldots, T^{* *}\left(m_{n}\right)\right)\right\rangle .
\end{aligned}
$$

For each $k=1, \ldots, n$ with $T^{* *}\left(m_{k}\right) \neq 0$, we take $\widetilde{d_{k}} \in Y$ with $\left\|\widetilde{d_{k}}\right\| \leq\left\|T^{* *}\left(m_{k}\right)\right\|$ such that

$$
\left|\widetilde{d_{k}}, g_{k}\right\rangle-\left\langle T^{* *}\left(m_{k}\right), g_{k}\right\rangle \mid<\frac{\delta}{16 n}\|g\|_{1}
$$


Define, for each $k=1, \ldots, n$,

$$
d_{k}:=\left\{\begin{array}{lll}
0 & \text { if } & T^{* *}\left(m_{k}\right)=0 \\
\widetilde{d_{k}} & \text { if } & T^{* *}\left(m_{k}\right) \neq 0 .
\end{array}\right.
$$

Moreover, for each $k=1, \ldots, n$, we choose $y_{k}$ in the closed unit ball of $Y$ with

$$
\operatorname{Re}\left\langle g_{k}, y_{k}\right\rangle \geq \frac{1}{2}\left\|\operatorname{Re}\left(g_{k}\right)\right\| \geq \frac{1}{4}\left\|g_{k}\right\| \text {. }
$$

Hence

$$
\begin{aligned}
\alpha & \geq \operatorname{Re}\left(\sum_{k=1}^{n}\left\langle g_{k}, d_{k}+\frac{\delta}{2} y_{k}\right\rangle\right) \geq \operatorname{Re}\left(\sum_{k=1}^{n}\left\langle g_{k}, d_{k}\right\rangle\right)+\frac{\delta}{2} \frac{1}{4} \sum_{k=1}^{n}\left\|g_{k}\right\| \\
& \geq \operatorname{Re}\left(\sum_{k=1}^{n}\left\langle g_{k}, T^{* *}\left(m_{k}\right)\right\rangle\right)-n \frac{\delta}{16 n}\|g\|_{1}+\frac{\delta}{8}\|g\|_{1} \\
& =\operatorname{Re}\left\langle g,\left(T^{* *}\left(m_{1}\right), \ldots, T^{* *}\left(m_{n}\right)\right)\right\rangle+\frac{\delta}{16}\|g\|_{1} \geq \alpha+\frac{\delta}{16}\|g\|_{1} .
\end{aligned}
$$

Therefore, we have that $0 \geq\|g\|_{1}$, so $g=0$ and we obtain a contradiction.

\section{ACKNOWLEDGMENT}

The authors thank Professor Arias de Reyna for his useful advice, especially concerning Example 2.1.

\section{REFERENCES}

[BCD] M. Basallote, M. D. Contreras and S. Díaz-Madrigal, Uniformly convexifying operators in classical Banach spaces, to appear in Bull. Austral. Math. Soc. CMP 99:11

[B] B. Beauzamy, Introduction to Banach spaces and their geometry, North-Holland, Amsterdam (1985). MR 88f:46021

[Be] S. F. Bellenot, Local reflexivity of normed spaces, operators, and Fréchet spaces, J. Func. Anal. 59 (1984) 1-11. MR 86a:46011

[D] D. W. Dean, The equation $L\left(E, X^{* *}\right)=L(E, X)^{* *}$ and the principle of local reflexivity, Proc. Amer. Math. Soc. 40 (1973) 146-148. MR 48:2735

[Di] J. Diestel, Sequences and series in Banach spaces, Grad. Texts in Math. n. 92, SpringerVerlag, New York, Berlin, Heidelberg (1984). MR 85i:46020

[GA] M. Gonzalez and A. Martínez-Abejón, Supertauberian operators and perturbations, Arch. Math. 64 (1995) 423-433. MR 96m:47022

[H1] S. Heinrich, Ultraproducts in Banach space theory, J. Reine Angew. Math. 313 (1980) 72-104. MR 82b:46013

[H2] S. Heinrich, Finite representability and super-ideals of operators, Dissertationes Mathematicae, Warszawa (1980). MR 81g:47047

Departamento de Matemática Aplicada II, Escuela Superior de Ingenieros, UniverSidad de Sevilla, Camino de los Descubrimientos, 41092 Sevilla, Spain

E-mail address: mabas@matinc.us.es

Departamento de Matemática Aplicada II, Escuela Superior de Ingenieros, UniverSidad de Sevilla, Camino de los Descubrimientos, 41092 Sevilla, Spain

E-mail address: madrigal@cica.es 